\title{
Hypertension at a glance
}

The Saudi Burden of Disease 2010 study showed that elevated blood pressure was the fourth-ranked risk factor for disability-adjusted life years (DALYs) in the Kingdom of Saudi Arabia in 2010. High blood pressure accounted for $8.7 \%$ and $6.4 \%$ of DALYs for males and females, respectively. Uncontrolled hypertension is strongly associated with higher risks of chronic diseases including atherosclerosis and aneurysm.

\section{Recent data from the Saudi Health}

Interview Survey (SHIS) show high rates of

hypertension in the Kingdom. An

individual is considered hypertensive if

their systolic blood pressure is $140 \mathrm{mmHg}$ or more, or if their diastolic blood pressure is $90 \mathrm{mmHg}$ or more, or if they have been diagnosed and are currently treated for hypertension. This large household survey showed that the prevalence of hypertension was $17.7 \%$ for males and $12.5 \%$ for females in 2013. The prevalence increased by age and was highest among those aged 65 or older (65.2\%). The prevalence of hypertension varied by region.

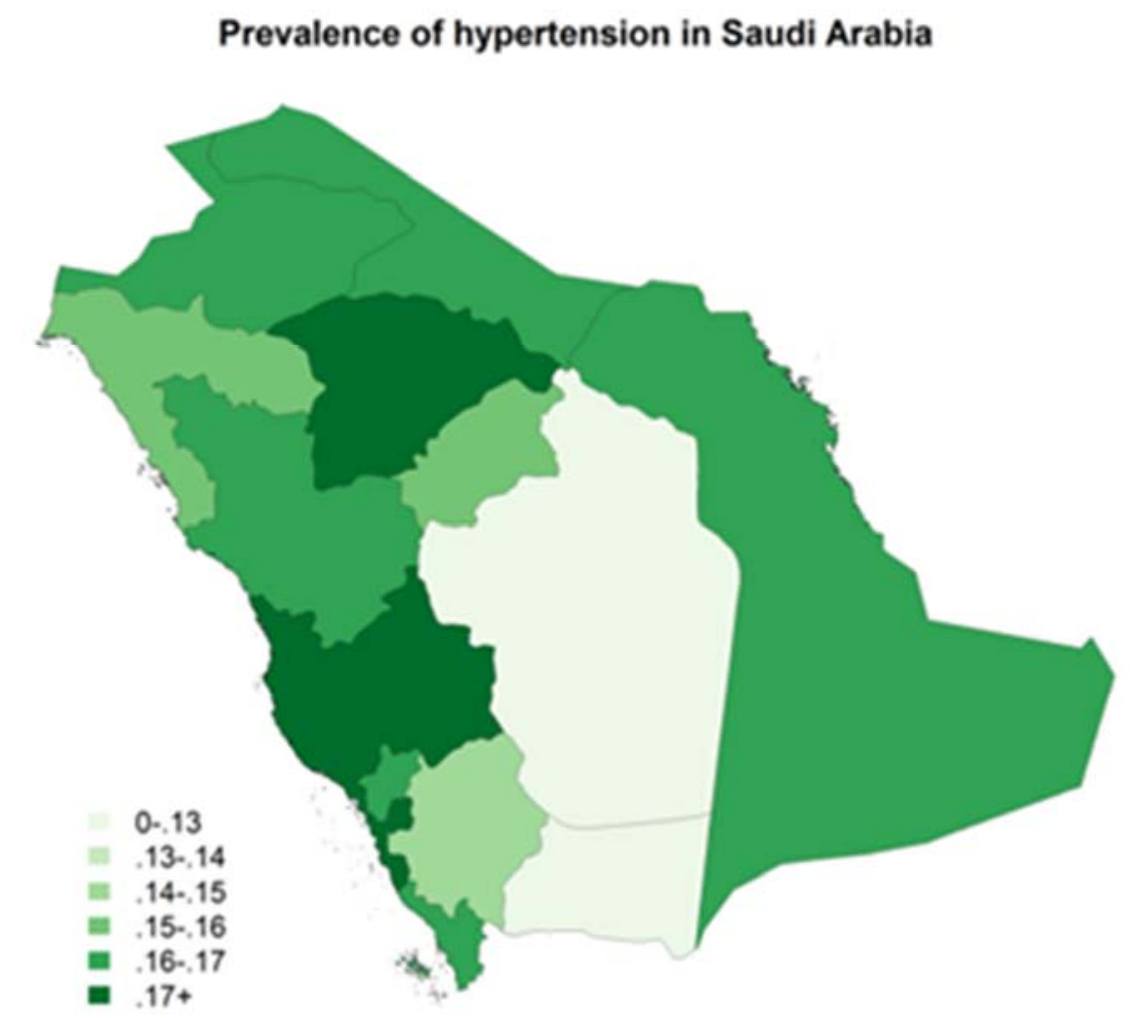


The results from the SHIS indicate that hypertension is associated with obesity and diabetes. Hypertension prevalence was $26.4 \%$ among those who were obese compared to $10.2 \%$ among those who were not obese. Furthermore, hypertension prevalence was $38.9 \%$ among those who were diabetic compared to $11.9 \%$ among those who were not diabetic.

The Saudi Ministry of Health utilizes a public health approach to address the burden of hypertension in the kingdom. The MOH programs include treatment and monitoring of those with high blood pressure levels and preventive programs. Many of the programs focus on the role of nutrition and physical activity in improving health and preventing and controlling chronic diseases. The scope of the Ministry's activities includes leadership, policy and guidelines development, surveillance, epidemiological and behavioral research, intervention development, technical assistance to regions and communities, training and education, communication, and partnership development.

\section{High blood pressure is a risk factor for:}

Heart disease and heart failure

Risk of atherosclerosis, a hardening of the arteries, is elevated in individuals with hypertension. This can lead to heart attacks or strokes.

\begin{tabular}{ll}
\hline Aneurysm & Aneurysms, or bulging blood vessels, can occur if \\
& blood pressure is elevated. If an aneurysm bursts, \\
& it can be fatal.
\end{tabular}

Organ damage Buildup of pressure in the blood vessels supplying organs such as the kidneys can prevent healthy organ functioning.

\begin{tabular}{ll}
\hline Vision loss & $\begin{array}{l}\text { Buildup of pressure in the blood vessels supplying } \\
\text { the eyes can result in vision loss. }\end{array}$ \\
\hline Metabolic syndrome & $\begin{array}{l}\text { Metabolic syndrome consists of elevated blood } \\
\text { pressure, insulin resistance, elevation of elements } \\
\text { of the blood associated with inflammation, and } \\
\text { elevation of clotting agents in the blood. }\end{array}$ \\
\end{tabular}

Difficulty with memory and comprehension Elevated blood pressure will impact one's ability to think and understand.

Source: Mayo Clinic, "High blood pressure (hypertension)," found at http://www.mayoclinic.com/health/high-bloodpressure/DS00100/DSECTION=complications, accessed on December 15, 2013. 
Hypertension is frequently referred to as a "silent killer" because unlike diabetes, for example, it has no symptoms and one can live for years without knowing one has it. For this reason, it is crucial to measure your blood pressure regularly, either at your doctor's office, a pharmacy, or using a home monitoring device. Fortunately, preventing and treating hypertension is straightforward.

Centers for Disease Control, "High blood pressure," found at http://www.cdc.gov/bloodpressure/, accessed on December 15, 2013.

\section{Take control and maintain a healthy blood pressure:}

Eat healthy foods and limit salt intake

Maintaining a healthy weight and limiting salt intake can both help to control blood pressure. Recommended sodium intake is 2,300 $\mathrm{mg} /$ day or less for young, healthy people, and an even lower level of $1,500 \mathrm{mg} /$ day is recommended for individuals over 50 years of age or those with hypertension, diabetes, or kidney disease.

Do not smoke
Exercise regularly and maintain a normal
BMI

Tobacco use accelerates the onset of atherosclerosis and causes damage to blood vessels.

A minimum of 30 minutes a day of physical activity is recommended to maintain a healthy weight. Being overweight or obese is strongly associated with high blood pressure.

Manage stress Anxiety and stress may also cause high blood pressure. Practice stress-reduction techniques and meditation.

Source: Mayo Clinic, "High blood pressure (hypertension)," found at http://www.mayoclinic.com/health/high-blood-pressure/DS00100/DSECTION=lifestyle-and-home-remedies, accessed on December 15, 2013. 
When measuring your blood pressure, use the guidelines outlined below. Systolic blood pressure is the pressure in your blood vessels during a heartbeat, and diastolic blood pressure is the pressure in your veins when your heart relaxes.

\section{Blood pressure levels}

\begin{tabular}{ll}
\hline Normal & Systolic: less than $120 \mathrm{mmHg}$ \\
& Diastolic: less than $80 \mathrm{mmHg}$ \\
\hline At risk (prehypertension) & Systolic: $120-139 \mathrm{mmHg}$ \\
\multirow{2}{*}{ High } & Diastolic: $80-89 \mathrm{mmHg}$ \\
& Systolic: $140 \mathrm{mmHg}$ or higher \\
\hline
\end{tabular}

Source: Centers for Disease Control, "High blood pressure," found at http://www.cdc.gov/bloodpressure/, accessed on December 15, 2013. 